\title{
A class of linear dynamical equations for a Banach space on time scales
}

Youling Feng*

"Correspondence: fengyl05@mails.ju.edu.cn Department of Applied Mathematics, Jilin University of Finance and Economics, Changchun, 130117, P.R. China

\begin{abstract}
In this paper we consider the existence and uniqueness of global solutions to linear dynamical equations for a Banach space on time scales from a new point of view. We characterize those linear dynamical equations for a Banach space whose existence and uniqueness of global solutions do not depend on concrete time scales.
\end{abstract}

MSC: 34G10; 34K30; 39A13

Keywords: time scales; linear dynamical equations; existence; uniqueness

\section{Introduction}

The calculus of time scales was introduced by Hilger in his PhD dissertation [1] in order to unify continuous and discrete analysis. In 2001, Bohner and Peterson published a comprehensive introduction to time scales [2]. Another book which consolidates further research in the area is [3]. The reader is referred to [1-9] for better understanding of time scales. We begin with introducing briefly some notation and terminologies.

We denote a time scale, which is a closed subset of the real numbers, by the symbol $\mathbb{T}$. Thus $\mathbb{R}$ and $\mathbb{Z}$, i.e., the real numbers and the integers, are the examples of time scales. The forward jump operator and backward jump operator $\sigma, \rho: \mathbb{T} \rightarrow \mathbb{T}$ and the graininess $\mu: \mathbb{T} \rightarrow[0, \infty)$ are defined by $\sigma(t):=\inf \{s \in \mathbb{T}: s>t\}, \rho(t):=\sup \{s \in \mathbb{T}: s<t\}$ and $\mu(t):=$ $\sigma(t)-t$ for $t \in \mathbb{T}$, respectively (supplemented by $\inf \emptyset=\sup \mathbb{T}$ and $\sup \emptyset=\inf \mathbb{T}$ ). A point $t \in \mathbb{T}$ is called right-dense, right-scattered, left-dense, left-scattered, if $\sigma(t)=t, \sigma(t)>t$, $\rho(t)=t, \rho(t)<t$ hold, respectively. The set $\mathbb{T}^{k}$ which is derived from the time scale $\mathbb{T}$ is defined as follows: If $\mathbb{T}$ has a left-scattered maximum $m$, then $\mathbb{T}^{k}=\mathbb{T}-\{m\}$. Otherwise, $\mathbb{T}^{k}=\mathbb{T}$.

Let $\mathcal{X}$ be a complex Banach space, $\mathcal{B}(\mathcal{X})$ be the class of all bounded linear operators on $\mathcal{X}$. A delta derivative for a function $f: \mathbb{T} \rightarrow \mathcal{X}$ was introduced by Hilger [7].

Let $t \in \mathbb{T}^{k}$. Then we define $f^{\Delta}(t)$ to be the element in $\mathcal{X}$ (provided it exists) with the property that given any $\varepsilon>0$, there is a neighborhood $U$ of $t$ (i.e., $U=(t-\delta, t+\delta) \cap \mathbb{T}$ for some $\delta>0)$ such that $\left\|[f(\sigma(t))-f(s)]-f^{\Delta}(t)[\sigma(t)-s]\right\| \leq \varepsilon|\sigma(t)-s|$ for all $s \in U(t, \delta)$. In this case, $f^{\Delta}(t)$ is called the delta (or Hilger) derivative of $f$ at $t$. Moreover, $f$ is said to be delta (or Hilger) differentiable on $\mathbb{T}^{k}$ if $f^{\Delta}(t)$ exists for all $t \in \mathbb{T}^{k}$.

This delta derivative is equal to $f^{\prime}$ (the usual derivative) if $\mathbb{T}=\mathbb{R}$, and it is equal to $\Delta f$ (the usual forward difference) if $\mathbb{T}=\mathbb{Z}$. So, the study of dynamical equations on time scales allows a simultaneous treatment of differential and difference equations. And this field has attracted many researchers' attention.

○2013 Feng; licensee Springer. This is an Open Access article distributed under the terms of the Creative Commons Attribution License (http://creativecommons.org/licenses/by/2.0), which permits unrestricted use, distribution, and reproduction in any medium, provided the original work is properly cited. 
Especially for linear dynamical equations in finite-dimensional spaces on time scales, a lot of results have been obtained (see [2-4, 10-15]). While for dynamical equations in Banach spaces on time scales, only a few results have been obtained (see $[7,16,17])$. In [7, 17], Hilger obtained the existence and uniqueness conditions for the global solutions to nonlinear and linear dynamical equations, while the conditions are closely dependent on the concrete time scales. It is natural to ask whether or not there exists a class of equations whose existence and uniqueness of global solutions do not depend on the concrete time scales.

In this paper, we consider the linear dynamical equation of the IVP

$$
\left\{\begin{array}{l}
y^{\Delta}(t)=A(t) y+g(t), \\
y\left(t_{0}\right)=y_{0}
\end{array}\right.
$$

where $\mathbb{T}$ is a time scale, the function $y$ is from $\mathbb{T}$ to $\mathcal{X}, y_{0} \in \mathcal{X}, g: \mathbb{T} \rightarrow \mathcal{X}$ is rd-continuous and $A: \mathbb{T} \rightarrow \mathcal{B}(\mathcal{X})$ is rd-continuous in the strong operator topology (SOT).

The purpose of this paper is to investigate those classes $\mathcal{A}$ of operators satisfying that for any time scale $\mathbb{T}$, if $V(A):=\{A(t) \in \mathcal{B}(\mathcal{X}): t \in \mathbb{T}\} \subseteq \mathcal{A}$, then equation (1) has exactly one global solution on the whole time scale. In order to state our main result, we first define a class $\mathfrak{U}$ of operators as

$$
\mathfrak{U}:=\left\{A \in \mathcal{B}(\mathcal{X}): \sigma_{1}(A) \cap \mathbb{R}^{-}=\emptyset\right\},
$$

where $\sigma_{1}(A):=\{\lambda \in \mathbb{C}: A-\lambda I$ is not invertible $\}$ denotes the spectrum of $A$ and $\mathbb{R}^{-}:=$ $(-\infty, 0)$.

The main result of this paper is the following theorem.

Theorem 1.1 The class $\mathfrak{U}$ is the largest one of those classes $\mathcal{A}$ ensuring that equation (1) has exactly one global solution for any time scale $\mathbb{T}$ and any rd-continuous $A: \mathbb{T} \rightarrow \mathcal{B}(\mathcal{X})$ with $V(A) \subseteq \mathcal{A}$.

From Theorem 1.1 we obtain the largest class of equations whose existence and uniqueness of global solutions do not depend on the concrete time scales. In Section 2, we make some preparations about time scales and show some needed lemmas. In Section 3, we give the proof of Theorem 1.1.

\section{Preparations}

Firstly, we introduce some needed definitions.

Definition 2.1 [2] A function $f: \mathbb{T} \rightarrow \mathcal{X}$ is said to be $r d$-continuous if it is continuous at right-dense points in $\mathbb{T}$ and its left-sided limits exist at left-dense points in $\mathbb{T}$. The set of all rd-continuous functions $f: \mathbb{T} \rightarrow \mathcal{X}$ will be denoted by $\mathrm{C}_{\mathrm{rd}}(\mathbb{T}, \mathcal{X})$.

Similarly, the set of all rd-continuous functions $f: \mathbb{T} \rightarrow \mathcal{B}(\mathcal{X})$ in the norm topology will be denoted by $\mathrm{C}_{\mathrm{rd}}(\mathbb{T}, \mathcal{B}(\mathcal{X}))$.

Definition 2.2 [2] A function $A: \mathbb{T} \rightarrow \mathcal{B}(\mathcal{X})$ is called regressive if $I+\mu(t) A(t)$ is invertible for all $t \in \mathbb{T}^{k}$. 
Definition 2.3 [2] Let $\mathbb{T}$ be a time scale and $\mathcal{X}$ be a Banach space. A function $f: \mathbb{T} \times \mathcal{X} \rightarrow$ $\mathcal{X}$ is said to be

(i) rd-continuous if $g(t)=f(t, x(t))$ is rd-continuous for any continuous function $x: \mathbb{T} \rightarrow \mathcal{X}$

(ii) regressive at $t \in \mathbb{T}^{k}$ if the mapping $i d+\mu(t) f(t, \cdot): \mathcal{X} \rightarrow \mathcal{X}$ is invertible (where $i d$ is the identity function), and $f$ is called regressive on $\mathbb{T}^{k}$ if $f$ is regressive at each $t \in \mathbb{T}^{k}$;

(iii) bounded on a set $S \subset \mathbb{T} \times \mathcal{X}$ if there exists a constant $M>0$ such that $\|f(t, x)\| \leq M$ for all $(t, x) \in S$.

Firstly, we introduce the existence and uniqueness theorem for the dynamical equation

$$
\left\{\begin{array}{l}
y^{\Delta}(t)=f(t, y) \\
y\left(t_{0}\right)=y_{0}
\end{array}\right.
$$

where $\mathbb{T}$ is a time scale, the function $y$ is from $\mathbb{T}$ to $\mathcal{X}, y_{0} \in \mathcal{X}, f(t, y)$ is a function from $\mathbb{T} \times \mathcal{X} \rightarrow \mathcal{X}$.

Lemma 2.4 [17] If $f(t, y)$ is regressive and rd-continuous on $\mathbb{T}^{k}$, then equation (2) has exactly one global solution on $\mathbb{T}$.

Especially for the linear form of equation (2), we obtain simpler conditions in the following lemma.

Lemma 2.5 Let $\mathbb{T}$ be some time scale and $f(t, y)=A(t) y+g(t)$, where $A: \mathbb{T} \rightarrow \mathcal{B}(\mathcal{X})$ and $y, g: \mathbb{T} \rightarrow \mathcal{X}$. Then the following two statements hold.

(i) The function $f(t, y)$ is regressive on $\mathbb{T}^{k}$ if and only if the function $A(t)$ is regressive on $\mathbb{T}^{k}$.

(ii) The function $f(t, y)$ is $r d$-continuous on $\mathbb{T}^{k}$ if and only if the function $A(t)$ is $r d$-continuous in the strong operator topology and $g(t)$ is $r d$-continuous on $\mathbb{T}^{k}$.

Proof (i) ' $\Longrightarrow$ '. Assume that $f(t, y)=A(t) y+g(t)$ is regressive on $\mathbb{T}^{k}$. Then, for any $t \in \mathbb{T}^{k}$, $b(t):=I+\mu(t) f(t, \cdot): \mathcal{X} \rightarrow \mathcal{X}$ is invertible. So $[I+\mu(t) A(t)]=b(t)-\mu(t) g(t)$ is invertible from $\mathcal{X}$ to $\mathcal{X}$ and therefore $A(t)$ is regressive on $\mathbb{T}^{k}$.

' $\Longleftarrow$ '. If the function $A(t)$ is regressive on $\mathbb{T}^{k}$, then for any $t \in \mathbb{T}^{k}, I+\mu(t) A(t)$ is invertible on $\mathcal{X}$. And then $[I+\mu(t) A(t)](\cdot)+\mu(t) g(t)$ is invertible from $\mathcal{X}$ to $\mathcal{X}$.

(ii) ' $\Longrightarrow$.' From Definition 2.3, it is easy to see that $g(t)$ is rd-continuous on $\mathbb{T}^{k}$ if we take the continuous function $y(t) \equiv 0$. Similarly, for any fixed $y \in \mathcal{X}$, let the function $y(t) \equiv y$ on $\mathbb{T}$, then $A(t) y+g(t)$ is also rd-continuous. Therefore $A(t) y=(A(t) y+g(t))-g(t)$ is rdcontinuous on $\mathbb{T}^{k}$. So the function $A(t)$ is rd-continuous in the strong operator topology.

' $\Longleftarrow$. For any continuous function $y(t)$ on $\mathbb{T}^{k}$, and any fixed right-dense point $t_{0} \in \mathbb{T}^{k}$, we have

$$
\left\|A(t) y(t)-A\left(t_{0}\right) y\left(t_{0}\right)\right\| \leq\left\|A(t)\left(y(t)-y\left(t_{0}\right)\right)\right\|+\left\|\left(A(t)-A\left(t_{0}\right)\right) y\left(t_{0}\right)\right\| .
$$

For $A(t)$ being rd-continuous in the strong operator topology, we have

$$
\lim _{t \rightarrow t_{0}}\left(A(t)-A\left(t_{0}\right)\right) y\left(t_{0}\right)=0 .
$$


So it suffices to show that for any $\varepsilon>0$, there exists $\delta>0$ such that for any $t$ with $\left|t-t_{0}\right|<$ $\delta$, we have $\left\|A(t) y(t)-A(t) y\left(t_{0}\right)\right\|<\varepsilon$.

If not, then there exist $\varepsilon_{0}>0$ and a sequence $\left\{t_{n}\right\}_{n=1}^{\infty}$ with $\lim _{n \rightarrow \infty} t_{n}=t_{0}$ such that

$$
\left\|A\left(t_{n}\right)\left(y\left(t_{n}\right)-y\left(t_{0}\right)\right)\right\|>\varepsilon_{0} \quad \text { for } n \geq 1 .
$$

For $A(t)$ being rd-continuous in the strong operator topology, we have

$$
\lim _{n \rightarrow \infty} A\left(t_{n}\right) y=A\left(t_{0}\right) y \quad \text { for any } y \in \mathcal{X} .
$$

So $\sup _{n \geq 1}\left\|A\left(t_{n}\right) y\right\|<\infty$ for any $y \in \mathcal{X}$. By the principle of uniform boundedness [18], there exists $M>0$ such that $\sup _{n \geq 1}\left\|A\left(t_{n}\right)\right\|<M$. And then $\lim _{n \rightarrow \infty}\left\|A\left(t_{n}\right)\left(y\left(t_{n}\right)-y\left(t_{0}\right)\right)\right\|=0$ from the continuity of $y(t)$. This is a contradiction to inequality (3).

So $f(t)=A(t) y(t)$ is continuous on right-dense points. Similarly, we can show that for left-dense points, the left-sided limits of $f(t)=A(t) y(t)$ exist.

It should be noticed that the proof of Lemma 2.5 is independent of special time scale features, except for the notion of rd-continuity at the end. We can obtain the following corollary easily by Lemma 2.4 and Lemma 2.5 .

Corollary 2.6 Let $\mathbb{T}$ be some time scale, $f(t, y)=A(t) y+g(t)$ be the right-hand side of equation (2). If the function $A(t)$ is regressive and $r d$-continuous in the strong operator topology on $\mathbb{T}^{k}, g(t)$ is rd-continuous on $\mathbb{T}^{k}$, then equation (2) has exactly one global solution on $\mathbb{T}$.

Remark 2.7 Corollary 2.6 implies the result for finite-dimensional spaces (see [2, Theorem 5.8]) and improves the conclusion for infinite-dimensional spaces (see [7, Section 6]) because the strong operator topology is equal to the norm topology in finite-dimensional spaces but weaker than the latter in infinite-dimensional spaces.

Therefore the condition 'strong operator topology' in (ii) of Lemma 2.5 cannot be replaced by 'norm topology'. In fact, we have the following counterexample.

Example 2.8 Let $L^{2}[0,1]$ be the space of Lebesgue square integrable functions on $[0,1]$, and let $\mathcal{B}\left(L^{2}[0,1]\right)$ be the class of all bounded linear operators on $L^{2}[0,1]$. For $t \in[0,1]$ and $x(s) \in L^{2}[0,1]$, define

$$
[A(t) x](s)= \begin{cases}x(s) & \text { if } s \in[0, t), \\ 0 & \text { if } s \in[t, 1] .\end{cases}
$$

Then $A:[0,1] \rightarrow \mathcal{B}\left(L^{2}[0,1]\right)$ is an operator-valued function. We first show that $A(t)$ is rd-continuous in strong operator topology.

Arbitrarily, choose $x \in L^{2}[0,1]$. For any $t_{0} \in(0,1)$ being right-dense, we have

$$
\lim _{t \rightarrow t_{0}}\left\|\left(A(t)-A\left(t_{0}\right)\right) x\right\|^{2}=\lim _{t \rightarrow t_{0}} \int_{\left[t_{0}, t\right) o r\left[t, t_{0}\right)}|x(s)|^{2} d s=0 .
$$

This implies that $A:[0,1] \rightarrow \mathcal{B}\left(L^{2}[0,1]\right)$ is continuous with respect to the SOT in the range space. On $\mathbb{T}=[0,1]$ the two notions of rd-continuity and continuity coincide. 
On the other hand, it is obvious that for any $t, t^{\prime} \in[0,1], t>t^{\prime}, A(t)-A\left(t^{\prime}\right)$ is an orthogonal projection on $L^{2}[0,1]$ with norm 1 . Therefore we conclude that $A(t)$ is not rd-continuous in the norm topology.

\section{Proof of the main result}

From Lemma 2.4 and Corollary 2.6, we can see that existence and uniqueness of the global solution to equation (1) depend closely on the regressivity of the operator-valued function $A(\cdot)$. Now we are going to give the proof of the main theorem.

Proof of Theorem 1.1 We give the proof by two steps.

Step $1 . \mathcal{U}$ is a class of operators which ensures that equation (1) has a unique solution for any time scale $\mathbb{T}$ and any $A: \mathbb{T} \rightarrow \mathcal{B}(\mathcal{X})$ with $V(A) \subseteq \mathcal{A}$.

We have the graininess $\mu: \mathbb{T} \rightarrow[0, \infty)$. If $V(A) \subseteq \mathcal{U}$, then $I+\mu(t) A(t)$ is invertible for any $t \in \mathbb{T}^{k}$. So $A(\cdot)$ is regressive on any time scale. Moreover, we know from equation (1) that $g(\cdot)$ is rd-continuous and $A(\cdot)$ is rd-continuous in the strong operator topology. From Corollary 2.6, equation (1) has a unique solution on the whole time scale.

Step 2. Suppose that $\mathfrak{M}$ is a class of operators with $\mathfrak{M} \nsubseteq \mathfrak{U}$ satisfying condition (i). We need only to prove that there exist a time scale $\mathbb{T}_{0}$ and a function $A$ from $\mathbb{T}_{0}$ to $\mathcal{B}(\mathcal{X})$ with $V(A) \subseteq \mathfrak{M}$ such that equation (1) either has no solution or has at least two solutions on $\mathbb{T}_{0}$.

Take $A_{0} \in \mathfrak{M} \backslash \mathfrak{U}$, then there exists $\lambda>0$ such that $-\lambda \in \sigma\left(A_{0}\right)$. We consider the following equation on $\mathbb{T}_{0}=\frac{1}{\lambda} \mathbb{Z}$ :

$$
\left\{\begin{array}{l}
y^{\Delta}(t)=A_{0} y \\
y\left(t_{0}\right)=y_{0}
\end{array}\right.
$$

where $y_{0} \neq 0$. Obviously, the solution to equation (4) is

$$
y\left(t+\frac{1}{\lambda}\right)=\frac{1}{\lambda}\left(A_{0}+\lambda I\right) y(t), \quad y\left(t_{0}\right)=y_{0} .
$$

It follows from $-\lambda \in \sigma\left(A_{0}\right)$ that $A_{0}+\lambda I$ is not invertible.

If $A_{0}+\lambda I$ is not injective, then $y\left(t_{0}-\frac{1}{\lambda}\right)$ is not unique.

If $A_{0}+\lambda I$ is not surjective, then we can choose $y_{0} \in \mathcal{X} \backslash \mathcal{R}\left(I+\frac{1}{\lambda} A_{0}\right)$ (where $\mathcal{R}\left(I+\frac{1}{\lambda} A_{0}\right)$ is the range of $\left.\left(I+\frac{1}{\lambda} A_{0}\right)\right)$. Hence $y\left(t_{0}-\frac{1}{\lambda}\right)$ does not exist. This completes the proof.

Remark 3.1 For $\mathcal{B}(\mathcal{X})$ being the matrix algebra $M_{n}(\mathbb{C})$, it is easy to see that the closure of $\mathfrak{U}$ is equal to $M_{n}(\mathbb{C})$. Hence we can deduce that $\mathfrak{U}$ is a very large class of operators in $M_{n}(\mathbb{C})$. We can also consider the closure and interior of the class $\mathfrak{U}$ in a Hilbert space in the future.

The authors declare that they have no competing interests.

\section{Acknowledgements}

The author gratefully acknowledges the invaluable advice and encouragement of Professor You Qing Ji. I would like to express my sincere thanks to the reviewers for their helpful comments and advice. Supported by NNSF of China $(11271150)$ and 'Twelfth Five-Year Plan' Science and Technology Research Project of the Education of Jilin Province $(2013215,2012186,2013373,2011113)$. 


\section{References}

1. Hilger, S: Ein maskettenkalkul mit anwendung auf zentrumsmannigfaltigkeiten. Dissertation, PhD, Univ. Wurzburg (1988)

2. Bohner, M, Peterson, A: Dynamic Equations on Time Scales. Birkhäuser, Boston (2001)

3. Bohner, M, Peterson, A: Advances in Dynamic Equations on Time Scales. Birkhäuser, Boston (2003)

4. Agarwal, RP, Bohner, M, O'Regan, D, Peterson, A: Dynamic equations on time scales: a survey. J. Comput. Appl. Math. $141(1-2), 1-26(2002)$

5. Akin, E, Bohner, M, Erbe, L, Peterson, A: Existence of bounded solutions for second order dynamic equations. J. Differ. Equ. Appl. 8(4), 389-401 (2002). In honor of Professor Lynn Erbe

6. Anderson, D, Peterson, A: Asymptotic properties of solutions of a $2 n$ th-order differential equation on a time scale. Math. Comput. Model. 32(5-6), 653-660 (2000)

7. Hilger, S: Analysis on measure chains - a unified approach to continuous and discrete calculus. Results Math. 18(1-2), 18-56 (1990)

8. Hilger, S: Matrix Lie theory and measure chains. J. Comput. Appl. Math. 141(1-2), 197-217 (2002)

9. Peterson, AC, Tisdell, CC: Boundedness and uniqueness of solutions to dynamic equations on time scales. J. Differ. Equ. Appl. 10(13-15), 1295-1306 (2004)

10. Agarwal, RP, Bohner, M, Shakhmurov, VB: Linear and nonlinear nonlocal boundary value problems for differential-operator equations. Appl. Anal. 85(6-7), 701-716 (2006)

11. Bohner, M, Saker, SH: Oscillation of second order half-linear dynamic equations on discrete time scales. Int. J. Differ. Equ. 1(2), 205-218 (2006)

12. DaCunha, JJ: Lyapunov stability and Floquet theory for nonautonomous linear dynamic systems on time scales Dissertation, PhD, Baylor University (2004)

13. Došlý, O, Hilger, S: A necessary and sufficient condition for oscillation of the Sturm-Liouville dynamic equation on time scales. J. Comput. Appl. Math. 141(1-2), 147-158 (2002)

14. Guseinov, GS, Zafer, A: Stability criteria for linear periodic impulsive Hamiltonian systems. J. Math. Anal. Appl. 335(2), 1195-1206 (2007)

15. Messer, KR: Linear dynamic equations on time scales. Dissertation, PhD, The University of Nebraska, Lincoln (2003)

16. Feng, YL: The solutions of linear dynamical equations in commutative Banach algebra on time scales. Acta Math. Sci. Ser. A Chin. Ed. 31A(2), 439-446 (2011)

17. Hilger, S: Differential and difference calculus - unified! In: Proceedings of the Second World Congress of Nonlinear Analysts, Part 5. (Athens, 1996), vol. 30, pp. 2683-2694 (1997)

18. Conway, JB: A Course in Functional Analysis, 2nd edn. Graduate Texts in Mathematics, vol. 96. Springer, New York (1990)

10.1186/1687-1847-2013-318

Cite this article as: Feng: A class of linear dynamical equations for a Banach space on time scales. Advances in Difference Equations 2013, 2013:318

\section{Submit your manuscript to a SpringerOpen ${ }^{\circ}$ journal and benefit from:}

- Convenient online submission

- Rigorous peer review

- Immediate publication on acceptance

- Open access: articles freely available online

- High visibility within the field

- Retaining the copyright to your article 